\title{
CAROTID REVASCULARIZATION CAN WE IMPROVE OUR EFFICIENCY?
}

Aliaño M, Gonzalez C, Paz D, Pintado C, Arastey S

Anaesthesia and Postsurgical Intensive Care, Complejo Hospitalario Universitario de Toledo, Spain

Introduction: The goal of this study is to determine the incidence of complications after carotid revascularization (stenting lendarterectomy) during the first $24 \mathrm{~h}$ and to identify risk factors which could help us to select patients for ambulatory procedures.

Materials and Methods: Observational retrospective research. Postsurgical patients undergoing carotid artery revascularization from 2012 to November 2016 were included in the study. The data was analyzed using a descriptive statistics and the Chi-square test. $P$ values less than 0.05 were considered statistically significant. All statistical analysis was conducted using SPSS 20.0 for Windows.

\section{Results and Discussion}

160 patients were included in the study (128 men, 32 women, mean age $71 \pm 9.5$ years, range $45-87$ years), treated for symptomatic $(n=99,62 \%)$ or asymptomatic $(n=61,38 \%)$, endarterectomy $(n=40,25 \%)$, stenting $(n=120,75 \%)$, urgent surgery $(n=6$, $3,8 \%)$, local anesthesia $(n=83,51,9 \%)$, general anesthesia $(n=77,48,1 \%)$.
47 patient ( $29,3 \%)$ had a total of 57 complications.

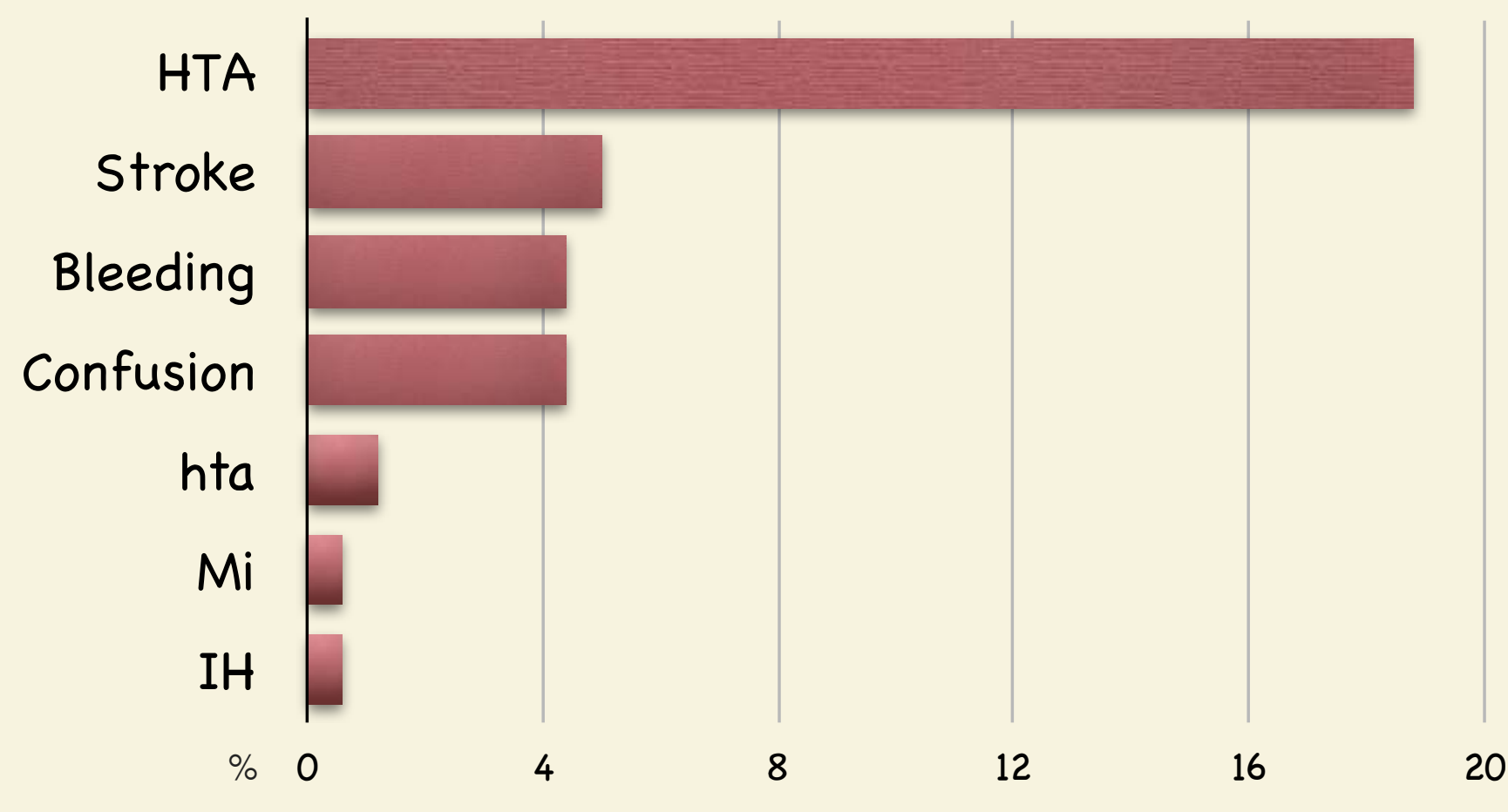

Endarterectomy showed a higher incidence of complications ( $47,5 \%$ vs $23,3 \%$ stent) P 0,04 . General anesthesia showed more complications than local anesthesia (40\% vs 19,75) p 0,04.

\section{$\int$ Conclusions}

This study shows a low incidence of fatal events.

The most common complication was hypertension.

Our patients undergoing carotid artery stenting may be safely discharged on the same day of surgery.

We recommend 6 hours of observation in the intensive care unit.

We suggest to provide local anesthesia whenever possible.

Our sample is not enough to determine whether the outpatient performance of endarterectomy is also a safe practice. 